\title{
Electrospun formulations of bevacizumab for sustained release in the eye
}

\author{
Ukrit Angkawinitwong ${ }^{1}$, Sahar Awwad ${ }^{1,2,3}$, \\ Peng T. Khaw ${ }^{2,3}$, Steve Brocchini ${ }^{1,2,3}$ and Gareth R. Williams ${ }^{1 *}$
}

${ }^{1}$ UCL School of Pharmacy, University College London, 29-39 Brunswick Square, London, WC1N 1AX, UK

${ }^{2}$ NIHR Biomedical Research Centre, Moorfields Eye Hospital, London, EC1V 9EL, UK

${ }^{3}$ UCL Institute of Ophthalmology, University College London, 11-43 Bath Street, London, EC1V 9EL, UK

*Corresponding author: g.williams@ucl.ac.uk 


\begin{abstract}
Medicines based on vascular endothelial growth factor (VEGF) neutralising antibodies such as bevacizumab have revolutionized the treatment of age related macular degeneration (AMD), a common blinding disease, and have great potential in preventing scarring after surgery or accelerating the healing of corneal injuries. However, at present, frequent invasive injections are required to deliver these antibodies. Such administration is uncomfortable for patients and expensive for health service providers. Much effort is thus focused on developing dosage forms that can be administered less frequently. Here we use electrospinning to prepare a solid form of bevacizumab designed for prolonged release while maintaining antibody stability. Electrospun fibers were prepared with bevacizumab encapsulated in the core, surrounded by a poly- $\varepsilon$-caprolactone sheath. The fibers were generated using aqueous bevacizumab solutions buffered at two different $\mathrm{pH}$ values: 6.2 (the $\mathrm{pH}$ of the commercial product; $\mathrm{F}_{\text {beva }}$ ) and 8.3 (the isoelectric point of bevacizumab; $\mathrm{F}_{\text {bevaP }}$ ). The fibers had smooth and cylindrical morphologies, with diameters of $c a .500 \mathrm{~nm}$. Both sets of bevacizumab loaded fibers gave sustained release profiles in an aqueous outflow model of the eye. $\mathrm{F}_{\text {beva }}$ displayed first order kinetics with $t_{1 / 2}$ of $11.4 \pm 4.4$ days, while $\mathrm{F}_{\text {bevaP }}$ comprises a zero-order reservoir type release system with $t_{1 / 2}$ of $52.9 \pm 14.8$ days. Both SDS-PAGE and surface plasmon resonance demonstrate that the bevacizumab in $F_{\text {bevap }}$ did not undergo degradation during fiber fabrication or release. In contrast, the antibody released from $F_{\text {beva }}$ had degraded, and failed to bind to VEGF. Our results demonstrate that $\mathrm{pH}$ control is crucial to maintain antibody stability during the fabrication of core/shell fibers and ensure release of functional protein.
\end{abstract}

Keywords: coaxial electrospinning, bevacizumab, controlled release system, coreshell fibers, anti-VEGF, poly-e-caprolactone 


\section{Statement of significance}

Bevacizumab is a potent protein drug which is highly effective in the treatment of degenerative conditions in the eye. To be effective, frequent injections into the eye are required, which is deeply unpleasant for patients and expensive for healthcare providers. Alternative methods of administration are thus greatly sought after to produce more effective medicines. In our work, we use the electrospinning technique to prepare fiber-based formulations loaded with bevacizumab. By careful control of the experimental parameters we are able to stabilize the protein during processing and ensure a constant rate of release of the protein over two months. These fibers could thus be used to reduce the frequency of dosing required, reducing cost and improving patient outcomes. 


\section{Introduction}

Antibody-based medicines targeted to vascular endothelial growth factor (VEGF) are widely used to treat a range of conditions in the eye. These include age related macular degeneration (AMD), the main cause of blindness in the elderly. AMD treatment involves direct injection of the antibody into the vitreous cavity of the eye (intravitreal injection). To be effective, intravitreal (IVT) injections are required every 4-8 weeks, which is considered too frequent as patients require treatment for many years [1], often decades. Further, IVT injections are invasive, carry some risk of retinal detachment and infection, and are also expensive to healthcare providers [2]. Other uses of antibodies in the eye include in aiding healing after injury or surgery. In the case of corneal injury, anti-VEGF antibodies can be applied to inhibit angiogenesis at the point of injury, thus helping to preserve sight. Surgery to make a small channel from the anterior chamber into the subconjunctival space is often required to reduce the intraocular pressure in patients with glaucoma, but frequently scarring occurs in the channel, causing the intraocular pressure to increase and glaucoma to progress. This scarring can be ameliorated through the use of antibodies. Thus, antibodies have enormous potential in treating conditions of the eye, but there remain a number of problems in their delivery: frequent injections to the eye are unpleasant, potentially dangerous, and expensive.

A slowly dissolving solid form of an antibody can potentially be used to slow antibody clearance from the vitreous cavity and subconjunctival space. There are a few clinically approved solid intravitreal ophthalmic drug implant technologies that have been developed for small molecules (e.g. steroids) [3], and some steroids are also administered as slow dissolving suspensions [4]. However, proteins are large, flexible molecules that are susceptible to aggregation, resulting in the loss of tertiary structure and function. Developing a solid dosage form designed for the slow dissolution and release of a stable functional antibody poses many challenges.

Bevacizumab is a monoclonal antibody that is widely used unlicensed in the clinic to treat AMD by IVT injection, as well as being potent when applied to the subconjunctival space to mediate healing after glaucoma surgery [5]. It also has a wide range of applications elsewhere in the body, and some sustained release formulations of bevacizumab have been reported (e.g. for cartilage tissue engineering [6] or brain tumor therapy [7]). There is a widespread ongoing research effort focused on 
developing longer acting dosage forms of bevacizumab for use in the eye, with systems based on for instance hydrogels $[8,9]$ and nanoparticles $[10,11]$ having been reported.

Electrospinning (ES) is a one-step 'top-down' process used to fabricate functional nanomaterials, and which has much promise in the development of new drug delivery systems (DDS) [12]. ES has been extensively applied in various facets of biomedical research such as tissue engineering, wound dressings, imaging and anticancer therapeutics $[13,14]$. In the process, a volatile solvent is first used to dissolve a polymer and a functional component(s). This solution is then loaded into a syringe and ejected through a metal needle (spinneret) towards a metal collector. There is an electrical potential difference applied between the spinneret and collector, typically with a high positive voltage at the spinneret and the collector being grounded. As the ES solution flows through the spinneret, the ejected droplets are stretched as the electrical force overcomes the surface tension of the liquid [15]. This deforms the spherical droplet into a conical shape at the spinneret (the Taylor cone). A polymer jet is ejected from the tip of this cone towards the collector, and the solvent is rapidly evaporated as the jet moves away from the spinneret. This yields solid non-woven fibers on the collector.

Compared to conventional encapsulation routes, ES involves electrical evaporation rather than using elevated temperatures to remove solvent. It can thus avoid any thermally-induced degradation of therapeutic proteins. Aqueous protein solutions need to be used for ES, however, because proteins will unfold or denature when exposed to most organic solvents in all but very dilute concentrations (of both protein and organic solvent) [16]. A modified form of ES called coaxial ES can be used to overcome this problem: this approach uses two concentric needles, one nested inside another, as the spinneret. Coaxial ES can in principle permit the encapsulation of bioactive proteins with minimal contact with any organic solvent. This is achieved by separately feeding an aqueous protein solution through the inner needle and the polymer solution (in a volatile organic solvent) through the outer needle. The coaxial method can also overcome other challenges associated with single-fluid ES, which can include an initial burst of release and the random distribution of encapsulated molecules in the fiber matrices $[17,18]$.

In this work, we have developed solid electrospun forms of bevacizumab which have the potential to be implanted in the eye, either in the vitreous cavity or subconjunctival space. Bevacizumab-loaded fibers were fabricated using coaxial 
electrospinning, encapsulating the antibody as the core material inside a poly- $\varepsilon$ caprolactone (PCL) shell. The $\mathrm{pH}$ used in the core solution was varied to determine its influence on bevacizumab stability and the release of protein from the fibers. The latter was probed using a flow chamber that mimics aqueous outflow in the subconjunctival space.

\section{Materials and methods}

\section{Materials}

Poly-e-caprolactone (PCL; 80 kDa), 2,2,2-trifluoroethanol (TFE), basic fuchsin, Dulbecco's phosphate buffered saline (PBS), fluorescein isothiocyanate isomer I (FITC), sodium bicarbonate, sodium carbonate monohydrate, Trizma ${ }^{\circledR}$ hydrochloride, Trizma ${ }^{\circledR}$ base, InstantBlue and human vascular endothelial growth factor $\left(V_{E G F} 165\right)$ were purchased from Sigma-Aldrich $(\mathrm{UK})$. Bevacizumab (Avastin ${ }^{\circledR}$, $25 \mathrm{mg} / \mathrm{mL}$, Genentech, US) was obtained from clinical donations that remained after an appropriate dose had been administered to patients. MicroBCA protein assays, Pierce $^{\mathrm{TM}}$ Silver Stain kits and PD-10 desalting columns were procured from Thermo Fisher Scientific (UK). Novex bis-tris 4-12\% SDS-PAGE gels, NuPAGE MOPS SDS running buffer, Novex Sharp pre-stained protein standard and NuPAGE LDS sample buffer (4X) were obtained from Life Technologies (UK). BIAcore consumables (including an amine coupling kit for ligand immobilisation which contained $\mathrm{N}$ hydroxysuccinimide (NHS) and 1-ethyl-3-(3-dimethylaminopropyl)carbodiimide (EDC), a regeneration scouting kit, and CM3 sensor chips) were sourced from GE Healthcare (UK). Vivaspin $6^{\circledR}$ centrifugal concentrators (molecular weight cut-off, MCWO $30 \mathrm{kDa}$ ) were purchased from VWR International (UK).

\section{Methods}

\section{FITC-bevacizumab conjugation}

FITC-conjugated bevacizumab (FITC-beva) was prepared to study its distribution in the fibers. Briefly, reconstituted bevacizumab solution $(1.4 \mathrm{mg} / \mathrm{mL}, 2.5$

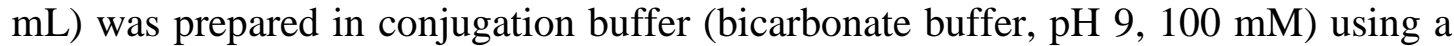
PD-10 desalting column to obtain the protein in the buffered solution $(1.0 \mathrm{mg} / \mathrm{mL}, 3.5$ $\mathrm{mL})$. FITC in conjugation buffer $(1 \mathrm{mg} / \mathrm{mL}, 175 \mu \mathrm{L})$ was incubated with the bevacizumab solution for $8 \mathrm{~h}$ at room temperature $\left(\mathrm{RT}, \sim 25^{\circ} \mathrm{C}\right)$ under gentle stirring and protected from light. The protein labeling ratio was 20:1 (1 mg protein: $0.05 \mathrm{mg}$ 
FITC). The reaction mixture was then purified using a PD-10 desalting column to eliminate unreacted FITC. The eluted fraction from the desalting column was subjected to size exclusion chromatography (SEC; Superose 12 10/300 GL column, Amersham Bioscience, US) using a mobile phase of PBS ( $\mathrm{pH} \mathrm{7.4)} \mathrm{at} \mathrm{a} \mathrm{flow} \mathrm{of} 1.0 \mathrm{~mL} / \mathrm{min}$ for 60 mins to purify the FITC-beva (see Supporting Information, Figure S1). The chromatographic system was equipped with Jasco (UK) HPLC systems including a pump (PU-980), AS-1555 autosampler and UV detector (UV-1570). Detection was performed at $280 \mathrm{~nm}$ (injection volume $300 \mu \mathrm{L}$ ). The HPLC systems was operated with Azur software version 5.0.10.0 (Kromatek, UK). The purified FITC-beva was centrifuged for 3 mins using a Vivaspin 6 centrifugal concentrator at $4000 \mathrm{rpm}$ and stored at $4{ }^{\circ} \mathrm{C}$ prior to further use.

SDS-PAGE

The FITC-beva conjugation and purification processes were monitored using SDS-PAGE. In brief, $15 \mu \mathrm{L}$ of each protein sample was mixed with $5 \mu \mathrm{L}$ of NuPAGE ${ }^{\circledR}$ LDS sample buffer (4X) and loaded on to Novex bis-tris 4-12\% SDS-PAGE gels. The gels were run at $200 \mathrm{~V}$ for 55 mins and analysed under a UV lamp at 254 / $365 \mathrm{~nm}$ (UVLS-28EL series UV Lamp, UVP, UK) to identify the fraction containing fluorescently labeled bevacizumab (see Figure S2). Subsequently, the gels were stained with InstantBlue for 35 mins and destained with water for 2 h (Figure S3).

\section{Preparation of bevacizumab loaded core shell fibers}

Bevacizumab-loaded core-shell fibers were formulated using different $\mathrm{pH}$ conditions for the protein core solution, to investigate the impact of this on the fibers' properties. Reconstituted Avastin ${ }^{\circledR}$ solutions (pH 6.2, $12.5 \mathrm{mg} / \mathrm{mL}, 1.0 \mathrm{~mL}$ ) and bevacizumab in Trizma ${ }^{\circledR}$ buffer $(\mathrm{pH} 8.3,12.5 \mathrm{mg} / \mathrm{mL}, 1.0 \mathrm{~mL}, 50 \mathrm{mM})$ were prepared prior to ES. The PCL shell (see Table 1) and bevacizumab core solutions were then separately loaded into 5.0 and $1.0 \mathrm{~mL}$ plastic syringes respectively. The syringes were mounted to feed a stainless steel coaxial spinneret (inner / outer needle internal diameters: $0.5 / 1.0 \mathrm{~mm}$ ). The spinneret was connected to a high voltage DC power supply (HCP 35-35000, FuG Elektronik, Germany). The solutions were ejected through the spinneret using two separate syringe pumps (78-9100C, Cole Parmer, UK) at constant flow rates. The fibers were collected on a grounded plate collector covered with aluminum foil. 
Coaxial ES was performed using parameters as given in Table 1. After the ES process, each electrospun fiber mat (ca. $500 \mathrm{mg}$ ) was stored at $2-8{ }^{\circ} \mathrm{C}$ before further characterization.

\section{Table 1}

Scanning electron microscopy (SEM)

An approximately $0.5 \times 0.5 \mathrm{~cm}^{2}$ section of each fiber sample was sputter coated with a $20 \mathrm{~nm}$ gold layer (Q150T coater, Quorum, UK) and analysed with a field emission scanning electron microscope (Quanta 200 instrument, FEI, USA) connected to a secondary Everheart-Thomley electron detector. The average fiber diameter was measured by using the ImageJ software version 1.49 (National Institutes of Health, USA) to determine the fiber dimensions at ca. 150 points of measurement in three SEM images.

Transmission electron microscopy (TEM)

A small amount of fibers was collected on a TEM grid by spinning directly onto the grid. The samples were then analysed using a field emission transmission electron microscope (Philips/FEI CM 120 Bio-Twin, FEI, USA).

\section{Digital microscopy}

Fibers were collected onto a glass slide and analysed using an inverted digital microscope (EVOS XL Cell Imaging System, Thermo Fisher Scientific, UK).

Differential scanning calorimetry (DSC)

DSC analysis was conducted using a Q2000 instrument (TA Instruments, USA). Samples (approximately $1 \mathrm{mg}$ ) were prepared in Tzero aluminum pans (T130425, TA instruments, USA) and sealed with pin-holed aluminum lids. The samples were heated from -70 to $150^{\circ} \mathrm{C}$ at a rate of $10^{\circ} \mathrm{C} / \mathrm{min}$. The DSC instrument was purged with nitrogen gas at a flow rate of $50 \mathrm{~mL} / \mathrm{min}$ throughout the measurements. The resultant data were analysed using the TA Universal Analysis software version 4.5A (TA Instruments, USA). 
Thermogravimetric analysis (TGA)

TGA analysis was performed using a Discovery TGA instrument (TA Instruments, USA). Samples (approximately $1 \mathrm{mg}$ ) were heated at $30{ }^{\circ} \mathrm{C}$ for $2 \mathrm{~h}$ in open aluminum pans. The instrument was purged with nitrogen gas at a flow of $25.0 \mathrm{~mL} / \mathrm{min}$ throughout. Data were analysed using the Trios software version 3.3.0.4055 (TA Instruments, USA).

Fourier-transform infrared spectroscopy (FTIR)

FTIR spectra of fiber samples (approximately $0.2 \times 0.2 \mathrm{~cm}^{2}$ ) were obtained using a Spectrum 100 spectrometer (Perkin Elmer, USA). The spectral data were analysed with the Essential FTIR v3.10.016 software (Operant LLC, USA). Data were collected over the wavenumber range from $650-4000 \mathrm{~cm}^{-1}$, with resolution $1 \mathrm{~cm}^{-1}$ and 4 scans obtained.

\section{Determination of protein distribution in core-shell fibers}

The purified FITC-beva solutions (see Supporting Information, Figure S2) were prepared in phosphate buffer ( $\mathrm{pH}$ 6.2) and Trizma ${ }^{\circledR}$ buffer ( $\mathrm{pH}$ 8.3) using a PD10 desalting column. Both solutions were then electrospun using the same method as described above. The resulting FITC-beva fibers were analysed using an inverted digital microscope, employing a GFP filtered-fluorescence mode in bright field, and TEM.

\section{Encapsulation efficiency}

Bevacizumab encapsulation efficiency was quantified using a modified method from the literature [19]. Approximately $20 \mathrm{mg}$ of protein-loaded fibers was extracted with $0.5 \% \mathrm{w} / \mathrm{v}$ sodium dodecyl sulfate (SDS) in 10/90 v/v dimethyl sulfoxide (DMSO): water with shaking for $2 \mathrm{~h}$ at RT. The supernatant was then collected and analysed using the microBCA assay. Encapsulation efficiency is presented as a percentage the theoretical protein loading.

In vitro release study

Release studies on the $\mathrm{F}_{\text {beva }}$ and $\mathrm{F}_{\text {bevap }}$ fibers were performed in an in-house flow rig model (see Figure 1). A cylindrical sample chamber with a diameter of $8.8 \mathrm{~mm}$, thickness of $3.27 \mathrm{~mm}$, and capacity of $200 \mu \mathrm{L}$ was employed for these studies. The rigs were rinsed, cleaned and dried prior to each experiment. The model was disassembled by removing the screws. Approximately $52.0 \mathrm{mg}\left(5.2 \times 7.2 \times 1.8 \mathrm{~mm}^{3}\right.$ of each sample 
was placed in each of the rigs, which were then reassembled. All rigs used in the experiment were placed in a pre-heated oil bath at $37^{\circ} \mathrm{C}$. An inlet portin the rigs allows a liquid flow similar to that in the front of the eye $(2.0 \mu \mathrm{L} / \mathrm{min})$ to be maintained $[20,21]$, and a constant flow of PBS ( $\mathrm{pH} 7.4$, at $37^{\circ} \mathrm{C}$ ) supplemented with sodium azide $(0.05 \%)$ was provided using a 16-channel Ismatec peristaltic pump (Michael Smith Engineers Ltd, UK). An outlet port is present to allow easy sample collection at predetermined time points, and experiments were performed over 3 weeks and 2 months for $F_{\text {beva }}$ and $\mathrm{F}_{\text {bevap }}$, respectively. The bevacizumab aliquots from each experiment were filtered with $0.22 \mu \mathrm{m}$ Millex-GP syringe filter units (Fisher Scientific, UK) prior to quantification using the MicroBCA assay. The results are presented as percent cumulative release:

$$
\% \text { Cumulative protein release }=\frac{M_{t}}{M} \quad 100
$$

Equation 1

where $M_{t}$ is the cumulative amount of bevacizumab released at time $\mathrm{t}$ and $M$ is the actual bevacizumab loading of each fiber. Further, the release profiles of the bevacizumab fibers were mathematically modeled using equations including the zeroorder, first-order, and Korsmeyer-Peppas equations [22]

\section{Figure 1}

\section{Silver staining SDS-PAGE}

Selected aliquots from the release experiments were assessed by SDS page to determine the protein integrity at each timepoint. The protocol from the Pierce ${ }^{\mathrm{TM}}$ Silver Stain Kit was followed when gels were analysed by silver staining. The solutions required were (i) 30\% ethanol : 10\% acetic acid in distilled water, (ii) 10\% ethanol, (iii) sensitiser working solution ( $50 \mu \mathrm{L}$ sensitiser with $25.0 \mathrm{~mL}$ water), (iv) working stain solution $(0.5 \mathrm{~mL}$ enhancer with $25.0 \mathrm{~mL}$ stain $)$, (v) working developer solution $(0.5 \mathrm{~mL}$ enhancer with $25.0 \mathrm{~mL}$ developer) and (vi) stop solution (5\% acetic acid in distilled water). After Coomasie blue staining and destaining, the gel was thoroughly washed twice with ultrapure water for 5 mins and then the gel was fixed with 30\% ethanol: $10 \%$ acetic acid solution for 15 mins. The gel was then washed twice with $10 \%$ ethanol and twice with water ( 5 mins per wash). After washing, the gel was incubated with the sensitiser working solution for $1 \mathrm{~min}$ and washed twice with ultrapure water (1 min each). The silver stain working solution was added to the gel, which was then incubated 
for 30 mins. The gel was washed twice with ultrapure water (1 min each) before the developer working solution was added and the gel incubated until protein bands appeared (usually within 2-15 mins). When the required band intensity was achieved, the stop solution (5\% acetic acid) was added for 10 mins and the final gel was washed with water.

\section{SPR Binding assay using Biacore}

Human VEGF165 (38 kDa) was immobilized on a CM3 chip to provide an immobilisation level of 50.4 RU. Briefly, the CM3 chip was first washed for $60 \mathrm{~s}$ with aqueous sodium hydroxide $(50.0 \mathrm{mM})$. The surface of the chip was then activated for $200 \mathrm{~s}$ with NHS $(300 \mu \mathrm{L}) / \mathrm{EDC}(300 \mu \mathrm{L})$, followed by immersion in a solution of $\operatorname{VEGF}(0.1 \mu \mathrm{g} / \mathrm{mL})$ in acetate buffer ( $\mathrm{pH} 5.5)$ for $150 \mathrm{~s}$. The active groups on the surface of the CM3 chip were subsequently deactivated with ethanolamine- $\mathrm{HCl}$ for $180 \mathrm{~s}$.

Samples were first quantified by microBCA to determine the protein concentrations, and the final concentrations to be tested were prepared in HBS-EPS buffer (containing 10.0 mM HEPES at pH 7.4, $150 \mathrm{mM} \mathrm{NaCl}, 3.0 \mathrm{mM}$ EDTA, and $0.005 \% \mathrm{P} 20$ ) for BIAcore. Binding assays were performed at $25{ }^{\circ} \mathrm{C}$ at a flow rate of $30.0 \mu \mathrm{L} / \mathrm{min}$, with association and disassociation times of 180 and $1200 \mathrm{~s}$ respectively and regeneration with glycine- $\mathrm{HCl}(\mathrm{pH} 2.0)$ for $30 \mathrm{~s}$. All data generated by BIAcore were evaluated with the BIAevaluation software version 2.1 (GE Healthcare, Sweden). The binding affinity was assessed by examining the relative response value (RU) obtained from the software.

Statistical analysis

Quantitative data are presented as mean \pm standard deviation, and have been statistically analysed using the Prism software version 6.0 (GraphPad, USA). Unpaired two-way T-tests were performed to calculate $p$ values for comparisons between two groups. A significant difference is defined when $p<0.05$. Half-lives $\left(t_{1 / 2}\right)$ were calculated according to the best fitting model in Prism. First-order kinetic rate constants ( $k$ ) were derived from the monoexponential curve and $t_{1 / 2} \mathrm{~s}$ calculated as $0.693 / k$. The rate constants $(k)$ of zero-order release profiles were calculated as concentration-time and $t_{1 / 2} \mathrm{~S}$ were obtained from the initial concentration $[\mathrm{A}]$ as $[\mathrm{A}] / 2 k$. 


\section{Results}

\section{Preparation and characterisation of bevacizumab-loaded core-shell fibers}

\section{Fiber morphology}

Bevacizumab (beva)-loaded PCL core-shell fibers were fabricated using the optimized parameters shown in Table 1. A 90\% v/v TFE: deionized water solution was found to be the optimum shell solvent to obtain reproducible core-shell fibers. The water co-solvent was used as this can lower the interfacial tension between the PCL shell and bevacizumab core fluids, facilitating the fabrication of core-shell fibers [23].

We also varied the $\mathrm{pH}$ of the protein core solution, as this has been reported to have an effect on the distribution of the encapsulated molecules in the fibers [24] and can influence the release mechanism and the stability of the protein guest. Fibers were first prepared in buffer at $\mathrm{pH} 6.2\left(\mathrm{~F}_{\text {beva }}\right)$, which is the $\mathrm{pH}$ of the reconstituted Avastin ${ }^{\circledR}$ solution [25]. A second set of fibers was also fabricated at $\mathrm{pH} 8.3\left(\mathrm{~F}_{\text {bevaP }}\right)$, the isoelectric point of bevacizumab [26,27]. SEM images of the fibers are shown in Figure 2a and b. Both sets of fibers show relatively smooth uniform structures, although there are

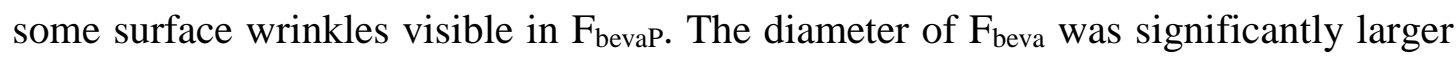
than that of $F_{\text {bevaP }}(520 \pm 120 \mathrm{~nm}$ vs $469 \pm 83 \mathrm{~nm}, p<0.05$;see Figure 2c and d).

The spatial location of bevacizumab in the fibers was probed by conjugating FITC to bevacizumab (Figure 2e and f). The $F_{\text {beva }}$ fibers display greater fluorescence intensity than the $\mathrm{F}_{\text {bevap }}$ materials, suggesting there is a greater tendency for the protein to migrate to the fiber shell when the core solution has a $\mathrm{pH}$ of 6.2. The TEM results (Figure $2 \mathrm{~g}$ and $\mathbf{h}$ ) indicate the $\mathrm{F}_{\text {bevap }}$ system has a clearly defined core/shell structure, while this is irregular and discontinuous with $\mathrm{F}_{\text {beva. }}$ The bevacizumab in $\mathrm{F}_{\text {beva }}(\mathrm{pH}$ 6.2) has a net positive charge, but in $\mathrm{F}_{\text {bevaP }}(\mathrm{pH}$ 8.3) it will be neutral. Clearly, the charge of the protein during ES has a profound effect on the protein distribution in the resultant fibers.

\section{Figure 2}

\section{Physical characterisation}

TGA analysis was used to analyze both sets of fibers after heating at $30{ }^{\circ} \mathrm{C}$ in isothermal mode. This temperature is lower than the melting point of PCL [28] and the 
degradation temperature of bevacizumab [29]. Therefore, degradation of the two materials is thus unlikely to occur during the TGA experiment. The thermograms (see Figure S4) show the mass loss was around $0.01 \%$ in both fiber sets, ten times lower than the sensitivity of the instrument $( \pm 0.1 \%$ mass change; Discovery TGA User Manual [30]) throughout the heating process. This means that the mass remained unchanged during heating, suggesting that both TFE and water from the shell and core compartments were completely evaporated during the ES process. Further physical characterisation of the bevacizumab fibers by DSC and FTIR (Figure S5 and S6) showed only the characteristic peaks of the PCL material, and the antibody signal was obscured. This is attributed to the low loading of the antibody in both fiber formulations, which results in the protein signals being overwhelmed by those from the polymer.

\section{Encapsulation efficiency and in vitro release study}

Prior to investigating the release kinetics, the encapsulation efficiencies of $F_{b e v a}$ and $F_{\text {bevap }}$ were determined. The theoretical loading was approximately $24 \mu \mathrm{g}$ bevacizumab in $1 \mathrm{mg}$ of the fabricated fibers. The encapsulation efficiency of $F_{b e v a}$ was $72.64 \pm 1.05 \%$ (approx. $908 \mu$ g bevacizumab), significantly higher than that observed for $\mathrm{F}_{\text {bevaP }}(63.15 \pm 0.30 \%$; approx. $789 \mu \mathrm{g}$ bevacizumab; $p<0.05)$.

Release studies were conducted in an in house in vitro flow rig model [31]. A constant flow rate of $2.0 \mu \mathrm{L} / \mathrm{min}$ was used to mimic the rate of aqueous turnover at the front of the eye [32,33]. The release media was supplemented with $0.05 \%$ sodium azide to prevent microbial growth. This apparatus was selected over the USP I or IV instruments because it more closely mimics the relevant parts of the eye. The microBCA assay was used to quantify the amount of bevacizumab in each aliquot collected and to calculate the cumulative release (see Figure 3). The dimensions of the fiber samples was equivalent to a $68 \mu \mathrm{L}$ volume, which is approximately three times smaller than the sample chamber.

\section{Figure 3}

Sustained release profiles were observed with both formulations throughout the study. $F_{\text {beva }}$ exhibited a $t_{1 / 2}$ of $11.4 \pm 4.4$ days with cumulative release of $60.6 \pm 7.3 \%$ (ca. $662 \mu \mathrm{g}$ ) of the actual loading over 19 days (Figure 3a). The F $\mathrm{bevap}_{\text {formulation }}$ 
displayed a much more prolonged release profile, with $t_{1 / 2}$ of $52.9 \pm 14.8$ days $(p=$ 0.0096). The amount of bevacizumab released from $F_{\text {bevap }}$ was $55.6 \pm 16.8 \%$ (ca. 439 $\mu \mathrm{g})$ after 60 days (Figure 3b). Conversely, the Avastin ${ }^{\circledR}$ solution was tested in the in vitro flow rig using the same dose ( $1.25 \mathrm{mg}$ in $200 \mu$ phosphate buffer) and displayed a much shorter $t_{1 / 2}$ of $\sim 4 \mathrm{~h}$ (see Figure S7)

Considering the profiles in more detail, it is clear that the concentration profile of $\mathrm{F}_{\text {beva }}$ is a biphasic release profile beginning with a burst phase over the first day (see Figure 3c). After $24 \mathrm{~h}$, we observed a bevacizumab concentration of $60.04 \pm 45.48$ $\mu \mathrm{g} / \mathrm{mL}$ (248 $\mu \mathrm{g}$, accounting for $24.6 \pm 19.3 \%$ of the total release). This is followed by a close-to-constant rate of release with the concentration ranging from 5.29-17.00 $\mu \mathrm{g} / \mathrm{mL}$ after 1 week of release and also over the remainder of the study period. Conversely, Fbevap displayed a continuous, monophasic, release profile over two months, with the concentration of the antibody ranging from $0.78 \pm 0.34$ to $7.38 \pm 6.67$ $\mu \mathrm{g} / \mathrm{mL}$ (Figure 3d).

The $t_{1 / 2}$ of bevacizumab clearance in a similar flow model has been found to be $1.2 \pm 0.1$ days in PBS, or $10.7 \pm 0.7$ days in simulated vitreal fluid [34]. Its clearance from the vitreous cavity in humans has been reported to have a $t_{1 / 2}$ of 4.9-10 days [3236]. Both the formulations prepared in this work are able to extend the residence time of the antibody considerably. As convective flow is responsible for drug elimination from the aqueous humor [37], the longer $t_{1 / 2}$ observed with the fibers over a bevacizumab solution suggests that the fibers can overcome rapid clearance by the aqueous flow in the eye. The bevacizumab-loaded fibers therefore have the potential to reduce very significantly the frequency of treatment required.

To elucidate the drug release mechanism, the release profiles were fitted with mathematical models. We hypothesized that drug diffusion would be a key mechanism contributing to the release of bevacizumab from the fibers. Given the slow hydrolytic degradation of PCL [28], it is unlikely that this polymer would undergo complete degradation in the conditions used to study release. Correlation coefficients $\left(\mathrm{R}^{2}\right)$, slopes after linear regression, release rate constants $(\mathrm{k})$ and other kinetic parameters obtained are given in Table 2 (kinetic fitting plots are provided in the Supporting Information, Figures S8-S13).

It is evident that the release profiles of both the $F_{b e v a}$ and $F_{b e v a p}$ fibers are governed by diffusion controlled mechanisms, as indicated by $\mathrm{R}^{2}$ being close to 1 for diffusion-release models. The kinetic profile of $F_{\text {beva }}$ was best fitted with a first-order 
equation $\left(\mathrm{R}^{2}=0.99\right)$. This is consistent with the results reported for most drug-loaded PCL fiber formulations, such as those containing tetracycline hydrochloride [38], bovine serum albumin [39], or alkaline phosphatase [19]. In contrast, the release profile from $F_{b e v a p}$ displayed zero-order kinetics, which is highly desirable in the design of controlled release systems. A zero-order release profile is very difficult to achieve with electrospun systems, because there is usually a significant burst release of drug in the initial stages of the process [40].

\section{Table 2}

To gain further understanding of the release behavior, we employed the Korsmeyer-Peppas (or Ritger-Peppas) equation [41]. The $\mathrm{n}$ exponent in this model gives information on the release phenomena from different geometrical dosage forms. It can be assumed that the release mechanism of polymeric systems follow Fick's law, in which drug diffusion is concentration-gradient dependent [42]. In the KorsmeyerPeppas model, ideal Fickian diffusion is observed when $n=0.5$ for a thin film, 0.45 for a cylindrical geometry, and 0.43 for a sphere; polymeric swelling is predominant when $\mathrm{n}$ is equal to $1.0,0.89$ and 0.85 , respectively.

The $n$ exponents from $F_{\text {beva }}$ and $F_{\text {bevaP }}$ were 0.60 and 0.82 , respectively. These values are between 0.45 and 0.89 , which is indicative of non-Fickian diffusion (given that the fibers are cylindrical), suggesting a more complex drug release mechanism is operational here. However, care should be taken with interpretation because the Korsmeyer-Peppas model is applicable only for systems with homogeneous drug distribution, which it is not the case in $\mathrm{F}_{\text {bevaP }}$ (as is clear from the TEM images). Srikar et al. [43] proposed that the desorption of solute from nanopores generated during the ES process is a key release mechanism for PCL nanofibers. This is consistent with the result from Gandhi's study [44] which demonstrated a similar mechanism for the release of protein from PCL fibers. We believe that this is also the case for both bevacizumab fiber formulations explored in this study, since our findings are wholly consistent with such a model. However, further investigation is required to unravel the details of the bevacizumab release mechanism from the PCL core-shell fibers. 


\section{In vitro stability of encapsulated bevacizumab}

The bevacizumab released from the in vitro flow rigs was evaluated by SDSPAGE with silver stain detection. Aliquots from days 1, 5, 10, 15 and 19 of the $F_{\text {beva }}$ release study, and those from days 1, 5, 10, 15, 20, 30, 40, and 60 from the $F_{\text {bevaP }}$ release study were evaluated (Figure 4). Bevacizumab showed a band at approximately 150 $\mathrm{kDa}$ (see control lane, Figure 4). Reconstituted Avastin ${ }^{\circledR}$ solution displayed aggregates (band > $160 \mathrm{kDa})$, intact bevacizumab $(150 \mathrm{kDa})$ and fragmented bevacizumab $(<160$ $\mathrm{kDa}$; lane 1). A trace amount of antibody fragment was also present at $50 \mathrm{kDa}$, along with other unidentified fragments. This indicates that the bevacizumab molecules underwent chemical degradation during incubation [45]. Similar degradation profiles were observed from all fractions released from $F_{\text {beva }}$ (see lane 2-6, Figure 4). The $F_{\text {beva }}$ fibers hence do not appear to stabilize bevacizumab. However, all the release fractions from the $\mathrm{F}_{\mathrm{bevaP}}$ formulation displayed only the band for the intact antibody at $150 \mathrm{kDa}$ (see lanes 7-14, Figure 4). There did not appear to be any protein fragmentation or aggregation here, and $\mathrm{F}_{\text {bevap }}$ thus successfully maintains the stability of the antibody.

\section{Figure 4}

Preliminary binding screening using surface plasmon resonance (SPR) was used to determine if the released bevacizumab would bind to immobilized VEGF (50.4 relative response $(\mathrm{RU})$ ). The response value was calculated from the sensorgram; a positive RU value means that bevacizumab underwent binding to VEGF, whereas a zero or negative value indicates no binding. Non-specific binding from the buffer (HPSEPS; negative control) should also give a RU value of zero.

The binding results are given in Figure 5. For $F_{\text {beva, }}$ positive RU values were observed in the samples collected up to day 10, but RU values close to zero or negative were obtained at day 15 and 19 (see Figure 5a). Thus, only the samples collected in the first 10 days of release retained their ability to bind VEGF. In contrast, all aliquots from the $\mathrm{F}_{\text {bevap }}$ study showed positive RUs (Figure 5b). Indeed, a quantitative binding analysis showed that the average RU remains approximately constant from the samples collected after 30, 40 and 60 days of $F_{\text {bevap }}$ release (see Figure 5c). The $F_{\text {bevaP }}$ formulation hence retains its anti-VEGF activity throughout the release period. The SPR results are in good agreement with the SDS-PAGE analysis in the case of $F_{\text {bevaP, }}$ 
confirming that the bevacizumab molecules remain intact over two months of release. However, the SPR and SDS-PAGE results for $\mathrm{F}_{\text {beva }}$ are more complicated as there is a combination of intact, aggregated and fragmented antibody in the collected aliquots (Figure 4), and the fragments could contribute to antibody binding in the SPR study if the binding site was not cleaved [45]. Even though some fractions from $F_{\text {beva }}$ show affinity, the inconsistent aggregation and fragmentation of bevacizumab visible in SDSPAGE raises concerns regarding this formulation.

\section{Figure 5}

\section{Discussion}

The results presented above clearly highlight that the $\mathrm{pH}$ of the protein solution used for ES has profound effects on the resultant fiber properties ( $\left.\mathrm{F}_{\text {beva }} c f \mathrm{~F}_{\text {bevaP }}\right) . \mathrm{pH}$ changes cause the net charge of bevacizumab to change, which affects the migration of the protein from the core to the shell fluid during ES. As the isoelectric point of bevacizumab is 8.3 [26,27], the protein in $\mathrm{F}_{\text {beva }}$ was positively charged at $\mathrm{pH}$ 6.2. There will be no net charge on the antibody when ES was conducted with Trizma buffer at $\mathrm{pH}$ 8.3. Tang et al. reported that the migration of macromolecules is driven by dielectrophoretic movement in the non-uniform electrical field generated during the ES process [24]. This means that any polarisable species may migrate toward the area where the strongest field is present, regardless of its electrostatic charge.

Coaxial ES is more complicated than single-fluid ES because a compound Taylor cone is developed. Luo and Edirisinghe [46] pointed out that charges can localize at the external interface between the shell polymer and air, creating an electrical field. This means that the outer cone will possess higher electric field intensity than the inner cone. Thus, it is expected that more charged macromolecules would accumulate in the shell than the core. In contrast, neutral moieties would be localized in the core. It can hence be hypothesized that bevacizumab will migrate to the shell in the spinning of $F_{b e v a}$ fibers, whereas in $F_{\text {bevap }}$ the protein would be completely encapsulated in the core.

The charged protein at $\mathrm{pH} 6.2$ can therefore migrate to the PCL fluid during Taylor cone development, and become immobilized there upon fiber solidification (see Figure 6a). This results in an uncontrolled distribution of bevacizumab throughout the 
$F_{\text {beva }}$ fibers, which may contribute to the release behavior of the fibers being similar to a monolithic system. In contrast, neutral bevacizumab at the $\mathrm{pI}$ was localised in the core of the $\mathrm{F}_{\text {bevap }}$ fibers (Figure 6b), resulting in zero-order release kinetics consistent with reservoir systems reported in the literature [47]. The results from this study thus show that zero-order release devices can be fabricated using coaxial ES by controlling the charge on the active ingredient being explored. This approach could be applied widely to other therapeutic payloads, where zero-order systems are highly desirable [40].

\section{Figure 6}

The protein distribution proposed for the $F_{\text {beva }}$ and $F_{\text {bevap }}$ fibers also explains the observed stabilities. Some positively charged bevacizumab in $\mathrm{F}_{\text {beva }}$ may be exposed to TFE in the shell fluid upon migration during Taylor cone formation. This could in turn lead to the protein becoming unfolded. Despite the possible unfolding mediated by TFE [48], however, the loss in structural integrity observed in this study is mostly ascribed to heavy-light chain fragmentation, as evidenced by the SDS-PAGE analysis. We assume that some bevacizumab molecules exposed to TFE were unfolded and immobilized in the polymer shell after ES. After elution and diffusion out of the fibers, the unfolded state of these proteins facilitated degradation during the release study. In contrast, the neutral bevacizumab remained in the core solution during $F_{\text {bevaP }}$ fabrication, thus precluding exposure to TFE (see Figure 6b). Therefore, the antibody remained intact after fabrication, and during the release study no fragmentation was detected in SDS-PAGE. Electrospinning at the pI of the antibody thus clearly improves the in-process stability of therapeutic proteins.

It should be noted that the encapsulation efficacy is another attribute influenced by the charge of the encapsulated bevacizumab. As previously described, charge generation on the polymer surface arising during ES contributes to the transportation of charged and uncharged species in the spinning fluid. Positively charged species are repelled from the inner surface of the needle and migrate toward the grounded electrode whereas neutral species remain in the bulk [15]. In our study, the positively charged protein in $\mathrm{F}_{\text {beva }}$ results in more bevacizumab being embedded in the polymer shell while the neutral species present during production of $\mathrm{F}_{\text {bevaP }}$ does not appear to be subject to such forces. This leads to higher encapsulation efficacies with $F_{\text {beva }}$ than $F_{\text {bevaP. }}$ However, further investigation is required to elucidate in more detail the effects of 
charge transport on the encapsulation efficacy, as this is a complex process and there is an interplay of several different factors which should be taken into account.

Our intent for this study was to prepare formulations for use intraocularly. The importance of the subconjunctiva part of the eye in this context relates to the clinical observation that the action of bevacizumab mediates healing after glaucoma surgery [5]. To reduce the intraocular pressure in patients with glaucoma, surgery is conducted to make a small channel from the anterior chamber (front of the eye) into the subconjunctival space, to allow the aqueous outflow to drain into the conjunctiva and the circulation. Often scarring occurs in this channel, blocking the aqueous outflow and causing the intraocular pressure to increase and glaucoma to progress. In previous work, we developed an implantable tablet form of bevacizumab that has been evaluated in the flow rig used in this study [49]. We found that the in vitro release results correlated with local pharmacokinetics in experimental glaucoma surgery, and the tablets were able to effectively mediate healing. The fibers prepared in this work have the potential to prolong bevacizumab release for a longer period of time than our existing formulation, and thus should result in further improvements to patient outcomes. Additionally, we have found that bevacizumab mediates healing after injury to the cornea, and placement of fibers near the point of injury in the cornea would help to maintain local bevacizumab concentrations, inhibiting angiogenesis within the cornea and preserving a patient's sight.

Our fibers thus have a wide range of potential applications as ocular implants. Since the fibers have very high surface areas, their degradation will be much more rapid that solid PCL implants (which last for $c a .3$ years in vivo). PCL is FDA-approved, and its degradation products are safe in humans. Thus, in the case of one-shot administration of the formulations (e.g. to prevent scarring after corneal injury) the fibres could safely be left in place to degrade after their therapeutic effects had been exhausted. In the case of multiple administrations, for instance for AMD treatment, then the surgeon could remove the currently placed implant and replace it with a fresh one. The in vivo performance of our materials will be explored in future work.

\section{Conclusions}

In this study sustained release systems for bevacizumab were fabricated using coaxial electrospinning. Core/shell systems were generated with an aqueous protein solution forming the core and PCL as the shell polymer. The $\mathrm{pH}$ of the core solution 
(6.2 or 8.3 (the pI of bevacizumab)) used for fabrication was varied and found to affect profoundly the release mechanism and stability of the protein in the fibers. While both sets of fibers were cylindrical in shape, those prepared at $\mathrm{pH} 6.2$ did not have a clear core/shell structure, and it is believed that some of the protein migrated to the shell during electrospinning. In contrast, the materials generated at $\mathrm{pH} 8.3$ had very distinct core and shell compartments. Sustained release profiles were seen from both sets of fibers. However, the release behavior of the fibers formed at the $\mathrm{pI}$ follows zero order kinetics, while those prepared at the lower $\mathrm{pH}$ show an initial burst release and first order kinetics. Moreover, electrospinning at the protein $\mathrm{pI}$ enhances its stability during release. Therefore, coaxial electrospinning shows great promise for the design of novel prolonged protein release devices. In the example system explored in this work, developing sustained release anti-VEGF formulations could profoundly improve patient health and wellbeing by reducing the requirement for frequent invasive dosing.

\section{Acknowledgments}

We thank David McCarthy and Kate Keen for SEM and TEM images, Dr. Asma Buanz for assistance with TGA experiments, and Mr John Frost for all his help to fabricating the rig models. S.A. gratefully acknowledges funding from the UCL Overseas Research Student Fund. We are further grateful for funding from: the National Institute of Health Research (NIHR) Biomedical Research Centre at Moorfields Eye Hospital NHS Foundation Trust and UCL Institute of Ophthalmology; Moorfields Special Trustees; the Helen Hamlyn Trust (in memory of Paul Hamlyn); the Medical Research Council; Fight for Sight; and, the Freemasons Grand Charity.

\section{References}

[1] S.D. Solomon, K. Lindsley, S.S. Vedula, M.G. Krzystolik, B.S. Hawkins, Antivascular endothelial growth factor for neovascular age-related macular degeneration, Cochrane Database Syst. Rev. 8 (2014) CD005139. doi:10.1002/14651858.CD005139.pub3.

[2] R.D. Jager, L.P. Aiello, S.C. Patel, E.T. Cunningham, Risks of intravitreous injection: a comprehensive review, Retina. 24 (2004) 676-98. http://www.ncbi.nlm.nih.gov/pubmed/15492621 (accessed May 17, 2017).

[3] Y.C. Kim, B. Chiang, X. Wu, M.R. Prausnitz, Ocular delivery of 
macromolecules, J. Control. Release. $190 \quad$ (2014) 172-181. doi:10.1016/j.jconrel.2014.06.043.

[4] A. Patel, K. Cholkar, V. Agrahari, A.K. Mitra, Ocular drug delivery systems: An overview., World J. Pharmacol. 2 (2013) 47-64. doi:10.5497/wjp.v2.i2.47.

[5] Z. Li, T. Bergen, S. Veire, I. Vel, H. Moreau, M. Dewerchin, P. Maudgal, T Zeyen, W Spileers, L Moons, I Stalmans, Inhibition of vascular endothelial growth factor reduces scar formation after glaucoma filtration surgery, Invest. Ophthalmol. Vis. Sci, 50 (2009) 5217-5225. doi: 10.1167/iovs.08-2662.

[6] M. Centola, F. Abbruzzese, C. Scotti, A. Barbero, G. Vadalà, V. Denaro, I. Martin, M. Trombetta, A. Rainer, A. Marsano, Scaffold-based delivery of a clinically relevant anti-angiogenic drug promotes the formation of in vivo stable cartilage. Tissue Eng. A 19 (2013) 1960-1971. doi: 10.1089/ten.TEA.2012.0455.

[7] C. Kutlu, A.S. Çakmak, M. Gümüşderelioğlu, Double-effective chitosan scaffold-PLGA nanoparticle system for brain tumour therapy: in vitro study, J. Microencapsul. 31 (2014) 700-707. doi: 10.3109/02652048.2014.913727.

[8] C.H. Wang, Y.S. Hwang, P.R. Chiang, C.R Shen, W.H. Hong, G.H. Hsiue, Extended release of bevacizumab by thermosensitive biodegradable and biocompatible hydrogel, Biomacromolecules 13 (2012) 40-48. doi: $10.1021 / \mathrm{bm} 2009558$.

[9] Y. Yu, L. C. M. Lau, A. C.-Y. LoY. Chau, Injectable chemically crosslinked hydrogel for the controlled release of bevacizumab in vitreous: A 6-month in vivo study, Trans. Vis. Sci.Technol. 4 (2015) 5. doi: 10.1167/tvst.4.2.5.

[10] F. Li, B. Hurley, Y. Liu, B. Leonard, M. Griffith, Controlled release of bevacizumab through nanospheres for extended treatment of age-related macular degeneration, Open Ophthalmol. J. 6 (2012) 54-58. doi: $10.2174 / 1874364101206010054$

[11] F. Sousa, A. Cruz, P. Fonte, I. Mendes Pinto, M.T. Neves-Petersen, B, Sarmento, A new paradigm for antiangiogenic therapy through controlled release of bevacizumab from PLGA nanoparticles, Sci. Rep. 7 (2017), 3736. doi:10.1038/s41598-017-03959-4.

[12] G.R. Williams, N.P. Chatterton, T. Nazir, D.G. Yu, L.M. Zhu, C.J. BrandfordWhite, Electrospun nanofibers in drug delivery: recent developments and perspectives, Ther. Deliv. 3 (2012) 515-533. doi:10.4155/tde.12.17. 
[13] A. Greiner, J.H. Wendorff, Electrospinning: a fascinating method for the preparation of ultrathin fibers., Angew. Chem. Int. Ed. Engl. 46 (2007) 5670703. doi:10.1002/anie.200604646.

[14] R. Sridhar, R. Lakshminarayanan, K. Madhaiyan, V. Amutha Barathi, K.H.C. Lim, S. Ramakrishna, Electrosprayed nanoparticles and electrospun nanofibers based on natural materials: applications in tissue regeneration, drug delivery and pharmaceuticals, Chem. Soc. Rev. 44 (2015) 790-814. doi:10.1039/C4CS00226A.

[15] A.L. Yarin, B. Pourdeyhimi, S. Ramakrishna, Electrospinning of micro-and nanofibers, in: Fundam. Appl. Micro-and Nanofibers, 1st ed., Cambridge University Press, Cambridge, 2014: pp. 179-261.

[16] U. Bilati, E. Allémann, E. Doelker, Strategic approaches for overcoming peptide and protein instability within biodegradable nano- and microparticles., Eur. J. Pharm. Biopharm. 59 (2005) 375-388. doi:10.1016/j.ejpb.2004.10.006.

[17] Y.K. Luu, K. Kim, B.S. Hsiao, B. Chu, M. Hadjiargyrou, Development of a nanostructured DNA delivery scaffold via electrospinning of PLGA and PLAPEG block copolymers, J. Control. Release. 89 (2003) 341-353. doi:10.1016/S0168-3659(03)00097-X.

[18] X.Y. Sun, L.R. Nobles, H.G. Börner, R.J. Spontak, Field-driven surface segregation of biofunctional species on electrospun PMMA/PEO microfibers, Macromol. Rapid Commun. $29 \quad$ (2008) 1455-1460. doi:10.1002/marc.200800163.

[19] W. Ji, F. Yang, J.J.J.P. van den Beucken, Z. Bian, M. Fan, Z. Chen, J.A. Jansen, Fibrous scaffolds loaded with protein prepared by blend or coaxial electrospinning., Acta Biomater. 6 (2010) 4199-207. doi:10.1016/j.actbio.2010.05.025.

[20] R.F. Brubaker, The flow of aqueous humor in the human eye., Trans. Am. Ophthalmol. Soc. $\quad 80 \quad$ 391-474. http://www.ncbi.nlm.nih.gov/pubmed/6763801 (accessed May 17, 2017).

[21] C.B. Toris, M.E. Yablonski, Y.L. Wang, C.B. Camras, Aqueous humor dynamics in the aging human eye., Am. J. Ophthalmol. 127 (1999) 407-412. doi:10.1016/S0002-9394(98)00436-X.

[22] S. Dash, P.N. Murthy, L. Nath, P. Chowdhury, Kinetic modeling on drug release from controlled drug delivery systems., Acta Pol. Pharm. 67 (2010) 217-23. 
doi:10.1016/S0928-0987(01)00095-1.

[23] H. Zhang, C. Zhao, Y. Zhao, G. Tang, X. Yuan, Electrospinning of ultrafine core/shell fibers for biomedical applications, Sci. China Chem. 53 (2010) 12461254. doi:10.1007/s11426-010-3180-3.

[24] C. Tang, A.E. Ozcam, B. Stout, S.A. Khan, Effect of $\mathrm{pH}$ on protein distribution in electrospun PVA/BSA composite nanofibers., Biomacromolecules. 13 (2012) 1269-78. doi:10.1021/bm2017146.

[25] Genentech, Avastin(R) (bevacizumab) [package insert], Genentech, Inc. (2004). https://www.accessdata.fda.gov/drugsatfda_docs/label/2009/125085s0169lbl.p df (accessed May 3, 2017).

[26] S. Kaja, J.D. Hilgenberg, E. Everett, S.E. Olitsky, J. Gossage, P. Koulen, Effects of dilution and prolonged storage with preservative in a polyethylene container on Bevacizumab (Avastin ${ }^{\mathrm{TM}}$ ) for topical delivery as a nasal spray in antihereditary hemorrhagic telangiectasia and related therapies., Hum. Antibodies. 20 (2011) 95-101. doi:10.3233/HAB-2011-0244.

[27] M. Vlčková, F. Kalman, M.A. Schwarz, Pharmaceutical applications of isoelectric focusing on microchip with imaged UV detection, J. Chromatogr. A. 1181 (2008) 145-152. doi:10.1016/j.chroma.2007.12.046.

[28] M.A. Woodruff, D.W. Hutmacher, The return of a forgotten polymerPolycaprolactone in the 21st century, Prog. Polym. Sci. 35 (2010) 1217-1256. doi:10.1016/j.progpolymsci.2010.04.002.

[29] H. Khalili, Disulfide-bridging PEGylation of antibody fragments, $\mathrm{PhD}$ thesis, University College London, 2012.

[30] TA Instruments, Discovery TGA User Manual.

[31] S. Awwad, A. Lockwood, S. Brocchini, P.T. Khaw, The PK-Eye: A novel in vitro ocular flow model for use in preclinical drug development, J. Pharm. Sci. 104 (2015) 3330-3342. doi:10.1002/jps.24480.

[32] T.U. Krohne, F.G. Holz, C.H. Meyer, Pharmacokinetics of intravitreally applied VEGF inhibitors, in: Springer International Publishing, 2016: pp. 139-148. doi:10.1007/978-3-319-24097-8_8.

[33] E. Moisseiev, M. Waisbourd, E. Ben-Artsi, E. Levinger, A. Barak, T. Daniels, K. Csaky, A. Loewenstein, I.S. Barequet, Pharmacokinetics of bevacizumab after topical and intravitreal administration in human eyes, Graefes Arch. Clin. Exp. Ophthalmol. 252 (2014) 331-7. doi:10.1007/s00417-013-2495-0. 
[34] C.H. Meyer, T.U. Krohne, F.G. Holz, Intraocular pharmacokinetics after a single intravitreal injection of $1.5 \mathrm{mg}$ versus $3.0 \mathrm{mg}$ of bevacizumab in humans, Retina. 31 (2011) 1877-1884. doi:10.1097/IAE.0b013e318217373c.

[35] Q. Zhu, F. Ziemssen, S. Henke-Fahle, O. Tatar, P. Szurman, S. Aisenbrey, N. Schneiderhan-Marra, X. Xu, S. Grisanti, Vitreous levels of Bevacizumab and vascular endothelial growth factor-A in patients with choroidal neovascularization, Ophthalmology $115 \quad$ (2008) 1750-1755.e1. doi:10.1016/j.ophtha.2008.04.023.

[36] P.M. Beer, S.J. Wong, A.M. Hammad, N.S. Falk, M.R. O’Malley, S. Khan, Vitreous levels of unbound bevacizumab and unbound vascular endothelial growth factor in two patients, Retina. 26 (2006) 871-876. doi:10.1097/01.iae.0000233327.68433.02.

[37] A. Urtti, Challenges and obstacles of ocular pharmacokinetics and drug delivery, Adv. Drug Deliv. Rev. 58 (2006) 1131-1135. doi:10.1016/j.addr.2006.07.027.

[38] H.J. Haroosh, Y. Dong, K. Lau, Tetracycline hydrochloride (TCH)-loaded drug carrier based on PLA:PCL nanofibre mats: experimental characterisation and release kinetics modelling, J. Mater. Sci. 49 (2014) 6270-6281. doi:10.1007/s10853-014-8352-7.

[39] H. Jiang, Y. Hu, Y. Li, P. Zhao, K. Zhu, W. Chen, A facile technique to prepare biodegradable coaxial electrospun nanofibers for controlled release of bioactive agents., J. Control. Release. $108 \quad$ (2005) 237-43. doi:10.1016/j.jconrel.2005.08.006.

[40] R. Siegel, M. Rathbone, Overview of controlled release mechanisms, in: Fundam. Appl. Control. Release Drug Deliv., 1st ed., Springer US, 2012: pp. $19-43$.

[41] P.L. Ritger, N.A. Peppas, A simple equation for description of solute release I. Fickian and non-fickian release from non-swellable devices in the form of slabs, spheres, cylinders or discs, J. Control. Release. 5 (1987) 23-36. doi:10.1016/0168-3659(87)90034-4.

[42] Y. Fu, W.J. Kao, Drug release kinetics and transport mechanisms of nondegradable and degradable polymeric delivery systems., Expert Opin. Drug Deliv. 7 (2010) 429-44. doi:10.1517/17425241003602259.

[43] R. Srikar, A.L. Yarin, C.M. Megaridis, A. V Bazilevsky, E. Kelley, Desorptionlimited mechanism of release from polymer nanofibers., Langmuir. 24 (2008) 
965-74. doi:10.1021/la702449k.

[44] M. Gandhi, R. Srikar, A.L. Yarin, C.M. Megaridis, R.A. Gemeinhart, Mechanistic examination of protein release from polymer nanofibers., Mol. Pharm. 6 (2009) 641-7. doi:10.1021/mp800160p.

[45] J. Vlasak, R. Ionescu, Fragmentation of monoclonal antibodies., MAbs. 3 (2011) 253-63. doi:10.4161/mabs.3.3.15608.

[46] C.J. Luo, M. Edirisinghe, Core-liquid-induced transition from coaxial electrospray to electrospinning of low-viscosity poly(lactide-co-glycolide) sheath solution, Macromolecules. $47 \quad$ (2014) 7930-7938. doi:10.1021/ma5016616.

[47] J. Siepmann, F. Siepmann, Mathematical modeling of drug delivery., Int. J. Pharm. 364 (2008) 328-43. doi:10.1016/j.ijpharm.2008.09.004.

[48] K. Gast, D. Zirwer, M. Müller-Frohne, G. Damaschun, Trifluoroethanol-induced conformational transitions of proteins: insights gained from the differences between alpha-lactalbumin and ribonuclease A., Protein Sci. 8 (1999) 625-34. doi:10.1110/ps.8.3.625.

[49] P. Khaw, S. Brocchini, H. Khalili, Composition for intraocular implantation of bevacizumab. US patent 9186414, 2010. 


\section{Tables}

Table 1 Coaxial ES parameters for the preparation of bevacizumab loaded core-shell fibers.

\begin{tabular}{ccc}
\hline Parameters & Fova & FovaP \\
\hline Core solution & $\begin{array}{c}\text { Reconstituted Avastin }{ }^{\circledR} \\
\text { solution in phosphate buffer } \\
(\mathrm{pH} 6.2,12.5 \mathrm{mg} / \mathrm{mL})\end{array}$ & $\begin{array}{c}\text { Bevacizumab in Trizma }{ }^{\circledR} \\
\text { buffer } \\
(\mathrm{pH} 8.3,12.5 \mathrm{mg} / \mathrm{mL})\end{array}$ \\
\hline Shell solution & $10 \%$ PCL in $90 \%$ v/v TFE: deionized water \\
\hline $\begin{array}{c}\text { Sheath/core flow rates } \\
\text { distance }\end{array}$ & $1.5: 0.3 \mathrm{~mL} / \mathrm{h}$ \\
\hline Voltage & $16 \mathrm{~cm}$ \\
\hline
\end{tabular}

Table 2 Kinetic parameters for bevacizumab release from the fibers.

\begin{tabular}{ccccccc}
\hline Sample & \multicolumn{3}{c}{ Release paramaters } & \multicolumn{3}{c}{ Korsmeyer-Peppas } \\
& $\boldsymbol{k}$ & $\boldsymbol{t}_{\mathbf{1} / \mathbf{2}}$ (days) & $\mathbf{R}^{\mathbf{2}}$ & $\mathbf{n}$ & $\mathbf{k}_{\mathbf{k p}}$ & $\mathbf{R}^{\mathbf{2}}$ \\
\hline $\mathrm{F}_{\text {beva }}$ & $0.13^{\mathrm{a}}$ & $5.9^{\mathrm{a}}$ & $0.99^{\mathrm{a}}$ & 0.60 & 13.30 & 0.99 \\
\hline $\mathrm{F}_{\text {bevaP }}$ & $0.84^{\mathrm{b}}$ & $62.5^{\mathrm{b}}$ & $0.99^{\mathrm{b}}$ & 0.82 & 1.73 & 0.99 \\
\hline
\end{tabular}




\section{Figure captions}

Figure 1 A schematic diagram illustrating the home-made dynamic flow cell apparatus used for dissolution studies. A dispensing pump continuously supplies a sample chamber with PBS buffer ( $\mathrm{pH} 7.4$, supplemented with $0.05 \%$ sodium azide) at flow rate of $2.0 \mu \mathrm{L} / \mathrm{min}$. The chamber is placed into a pre-heated oil bath to maintain the temperature at $37^{\circ} \mathrm{C}$. Aliquots were removed from the outlet tube at predetermined time points for further analysis.

Figure 2 SEM images of a) bevacizumab-PCL core/shell fibers spun with sodium phosphate buffer, pH $6.2\left(\mathrm{~F}_{\text {beva }}\right)$ and b) bevacizumab-PCL core/shell fibers spun with Trizma buffer at $\mathrm{pH} 8.3\left(\mathrm{~F}_{\text {bevaP }}\right)$, together with the size distributions of $\left.\mathbf{c}\right) \mathrm{F}_{\text {beva }}$ and $\mathbf{d}$ ) $F_{\text {bevaP }}\left(\right.$ mean $\pm S D$ ), and fluorescent microscopy images of e) $F_{\text {beva }}$ and $f$ ) $F_{\text {bevaP }}$ prepared with FITC-beva. TEM images illustrating the internal structures are also given for $\mathbf{g}$ ) $F_{\text {beva }}$ and $\left.\mathbf{h}\right) \mathrm{F}_{\text {bevap }}$ fibers. Arrows indicate the encapsulated bevacizumab in the fibers.

Figure 3 Cumulative release profiles of $\mathbf{a}) \mathrm{F}_{\text {beva }}(n=3)$ and $\left.\mathbf{b}\right) \mathrm{F}_{\text {bevaP }}(\mathrm{n}=3)$ in a rig model mimicking the aqueous turnover of the anterior segment of the human eye. The inset in b) shows the release profile in the first 5 days of the release experiment of $F_{\text {bevaP. }}$. Also depicted are concentration vs time profiles of c) $F_{\text {beva }}$ and d) $F_{\text {bevap. Data are shown as }}$ mean \pm SD.

Figure 4 Silver staining of a Novex Bis-Tris 4-12\% gel loaded with bevacizumab collected from release studies (lane 1-14). Lane M: molecular weight standard; $\underline{\text { Control: }}$ : freshly-prepared Avastin ${ }^{\circledR}$ solution; lane 1: Avastin ${ }^{\circledR}$ solution after incubation in the flow rig; lanes 2-6: F Feva at day 1, 5, 10, 15 and 19 respectively; and lanes 7-14: FbevaP at day $1,5,10,15,20,30,40$ and 60 respectively.

Figure 5 SPR results showing VEGF binding by bevacizumab, compared with HPSEPS buffer (negative control). Preliminary data are shown for bevacizumab released from a) $F_{\text {beva }}(n=1)$ and b) $F_{\text {bevaP }}(n=1)$, together with $\left.\mathbf{c}\right)$ a detailed SPR binding analysis of aliquots collected from $\mathrm{F}_{\text {bevaP }}$ at day 40,50 and 60, with the concentration of bevacizumab quantified by the MicroBCA assay $(n=3$; data presented as mean \pm SD ) .

Figure 6 A schematic diagram illustrating the effect of bevacizumab migration during electrospinning on the fiber properties. a) During the fabrication of $F_{b e v a}$, the protein carries a net positive charge at $\mathrm{pH} 6.2$; this causes it to migrate uncontrollably from the aqueous core to the polymer shell and become unfolded upon exposure to TFE. The resultant distribution of both intact bevacizumab and unfolded bevacizumab within the $\mathrm{F}_{\text {beva }}$ core and shell contributes to the first-order release behavior observed. b) In contrast, the uncharged protein remains in the aqueous core for $F_{\text {bevaP }}$ (prepared at the $\mathrm{pI}, \mathrm{pH}$ 8.3), which leads to zero-order release and intact bevacizumab. 